\title{
Modelling axial dispersion of granular material in inclined rotating cylinders with bulk flow
}

\author{
J. R. Third · D. M. Scott • G. Lu • C. R. Müller
}

Received: 22 May 2014 / Published online: 8 January 2015

(C) Springer-Verlag Berlin Heidelberg 2015

\begin{abstract}
The axial dispersion of approximately monosized particles in rolling mode in rotating cylinders with bulk flow is examined using a Monte Carlo model and discrete element method (DEM) simulations. The Monte Carlo model predicts that the mean square displacement relative to the mean axial displacement of the bed undergoes oscillations in time. The nature of these oscillations depends on the fill level of the cylinder and the extent of particle mixing during avalanches. When the cylinder is half full the Monte Carlo model predicts undamped oscillations, whereas a filling fraction of 0.26 produces oscillations whose amplitude decreases with time. If mixing during avalanches is assumed to be perfect then the oscillations occur about a linear increase with time. In contrast, if it is assumed that the particles do not mix during avalanching, the oscillations occur about an increase with time which has a gradient which increases with time. There is good qualitative agreement between the Monte Carlo model with perfect mixing and the DEM when the filling fraction is 0.26 . For a filling fraction of 0.5 the DEM data show oscillations about a faster than linear increase with time.
\end{abstract}

Keywords Axial dispersion - Rotating cylinders

J. R. Third · G. Lu · C. R. Müller (凶)

Department of Mechanical and Process Engineering, Institute of Energy Technology, ETH Zürich, Leonhardstraße 21, 8092 Zürich, Switzerland

e-mail: muelchri@ethz.ch

D. M. Scott

Department of Chemical Engineering and Biotechnology,

University of Cambridge, Pembroke Street,

CB2 3RA Cambridge, UK

\section{Introduction}

Despite their omnipresence in nature and their relevance for many practical applications, there is still no governing macroscopic equation system for granular materials [1]. Our comparatively limited fundamental understanding of such systems can be attributed to the inherent complexity of granular systems [2], and to the difficulty of obtaining experimental measurements of sufficient temporal and spatial resolution due to their optical opacity [3]. Furthermore, depending on the boundary conditions, packing density and excitation mechanism, granular materials can possess properties which are otherwise encountered in solid, liquid or gaseous systems $[1,4,5]$.

A particular experimental configuration that has allowed the probing of different phenomena related to the dynamics of granular systems, such as mixing, segregation or avalanching, is the rotating cylinder [2,6-9]. In particular, axial diffusion in granular systems (often also referred to as axial dispersion) has attracted significant attention $[10,11]$. An understanding of dispersion in rotating cylinders on a fundamental level is of significant practical relevance since it controls the residence time distribution (RTD) of the particles and, in turn, product homogeneity.

In this paper, the motion of the particles is taken to be rolling, with the bed surface flat in a cross section of the cylinder. The forms of motion and transitions have been reviewed by Mellmann [12]. A particle moves with the cylinder in rigid body rotation, with little or no change in axial position, until it reaches the free surface of the bed of particles. Then it falls down the free surface as part of an avalanche, until it is reabsorbed into the rigid body rotation. During the avalanching, collisions between particles can produce axial movement, leading to axial dispersion. Such axial dispersion will be referred to as collisional; Hehl et al. [13] and Third 
and Müller [14] have discussed an analogy with the Galton board. Based on experimental measurements and numerical studies it is now generally accepted that dispersion in horizontally rotating cylinders follows Ficks law [15-18], i.e. the mean square deviation of particle position increases linearly with time:

$\left\langle(z(t)-z(0))^{2}\right\rangle=2 D_{\mathrm{ax}} t$

where $z$ is axial position, $D_{\mathrm{ax}}$ is a constant dispersion coefficient and $t$ is time. However, as recently demonstrated analytically by Christov and Stone [19], the initial width of the diffusing particle pulse has to be considered when calculating the axial dispersion coefficient from concentration profiles. Nonetheless, dispersion in rotating cylinders is yet far from well understood since both numerical and experimental studies have predominantly relied on ideal systems, i.e. cylinders with the axis of rotation horizontal and filled with monodisperse particles. For horizontal cylinders there is no bulk axial motion. However, in practical systems, rotating drums are inclined, inducing a bulk particle motion. In the work that has considered bulk flow through the cylinder particle motion has been modelled based on the geometric models of Pickering et al. [20], Saeman [21], Vàhl and Kingma [22] and Kramers and Croockewit [23]. In these models, particles are assumed to move as described previously, except that during the avalanching a particle has significant axial movement. It is assumed that the surface of the bed is locally flat and that in an avalanche particles travel in straight lines inclined at the angle of repose. When the surface of the bed has an inclination in the axial direction, particles falling in an avalanche down the line of steepest descent are absorbed into the rigid body rotation at different axial positions, which gives rise to axial dispersion, which will be referred to as geometric axial dispersion. For a horizontal bed there is no geometric axial dispersion. Rogers and Gardner [24] used a Monte Carlo method to calculate RTDs to compare to experimental data. Probability distributions were used to choose how far each particle travelled in an avalanche, and how the direction of its path deviated from that given by the line of steepest descent. Rogers and Gardner concluded that collisional dispersion was more important than geometric dispersion for RTDs. Das Gupta et al. [16] and Rao et al. [25] measured RTDs and used a Monte Carlo method to calculate RTDs to compare to their own data, and those of Hehl et al. [13]. They concluded that collisional axial dispersion was important in fitting RTDs.

In this work we report results from Monte Carlo and Lagrangian modelling of axial dispersion of approximately monosized particles in an inclined rotating cylinder with bulk flow, in the special case where the granular bed is of uniform depth. Beds of uniform depth are studied for simplicity; they can be achieved throughout the cylinder by the use of an annu- lar dam at the discharge end, and whatever the end condition approximately uniform depth occurs sufficiently far from the discharge end. The conditions for uniform bed depth can be found from the models of Pickering et al. [20], Saeman [21], Vàhl and Kingma [22] and Kramers and Croockewit [23]. Experimental data on the influence of an annular dam on bed depth have been given by Scott et al. [26]. The cylinder is rotating fast enough for continuous avalanching to occur on the free surface of the bed (rolling regime), but slowly enough for the bed surface to be flat. It will be found that at short times, and particularly for larger angles of inclination of the cylinder, geometric axial dispersion leads to oscillations in the mean squared axial displacement of particles.

Rapaport [27] has shown that in DEM simulations of particle dynamics in horizontal rotating cylinders, using periodic boundary conditions (PBCs), bulk displacement of the bed can occur, which affects axial dispersion. Third et al. [28] have shown that in this case axial dispersion occurs independently of the bulk motion, and that a measure of the magnitude of axial dispersion can be obtained by considering the mean square deviation relative to the mean axial displacement of the bed, RMSD,

$R M S D=\left\langle(z(t)-z(0))^{2}\right\rangle-\langle(z(t)-z(0))\rangle^{2}$

$R M S D$ is the second central moment of the distribution of axial displacements. It therefore provides useful information about the width of the RTD, and thus product composition, for cylinders with bulk flow. Recent measurements of axial dispersion in pilot plant rotating cylinders have been made by Gao et al. [29]. Campbell [30] has shown that the underlying behaviour of dispersion in shear flows follows Fick's law so long as the bulk motion is accounted for correctly. However, since both geometric and collisional dispersion contribute to the rate of axial dispersion within rotating cylinders, it is important to consider a measure which takes both effects into account. Therefore $R M S D$, which does take into account both geometric and collisional dispersion, will be used in the present work to quantify axial dispersion.

The developed Monte Carlo model of particle motion, using the geometry of the bed together with particle motion in sequential rigid body rotation and avalanching will be presented in Sect. 2. The results show that for short times the RMSD can behave as an oscillation about an increase with time. It is emphasised that this model is illustrative of the consequence of the assumptions about particle motion: it is not intended to provide a realistic, accurate description of particle motion. In Sect. 3 DEM calculations, which are intended to provide a more realistic description of particle motion, are presented. These calculations show that the behaviour of RMSD depends critically on the fill level. For short times, RMSD displays an oscillation about a linear increase with time at low fill, whereas for a half-full cylinder 
RMSD oscillates about a line that increases faster than linearly. In contrast, if it is assumed that the particles do not mix during avalanching, the oscillations occur about an increase with time which has a gradient which increases with time.

\section{Monte Carlo Model}

Monte Carlo models of rotating cylinders, which differ in detail, have been described in [16,24,31-33]. The method used here will now be described, and notation defined. The method is essentially the same as those Monte Carlo methods described in [16,24,31-33].

A cylinder of radius $R$, with axis inclined at angle $\beta$ to the horizontal, rotates about its axis at rotational speed $n$ (rps), and a bed of particles flows through the cylinder. The size of the particles is assumed to be negligible compared to the size of the cylinder. The surface of the bed is assumed to be flat, and the bed is of uniform depth $h$. A cross section of the cylinder is shown in Fig. 1. The angle of repose of the particles is $\gamma$, and it is assumed that the steepest slope in the surface of the bed is $\gamma$. Then the angle $\psi$, defined in Fig. 1, is given by

$\cos \psi=\frac{\cos \gamma}{\cos \beta}$

The fill, that is the fraction of the cross section occupied by the granular bed, is given by

fill $=\frac{1}{\pi}(\phi-\sin \phi \cos \phi)$

where

$\phi=\cos ^{-1}\left(1-\frac{h}{R}\right)$

is defined on Fig. 1. The surface of the bed is shown in Fig. 2, where the right is lower than the left. A particle leaves the region of rigid body rotation, and appears on the surface at $A$. The particle is assumed to travel in an avalanche along

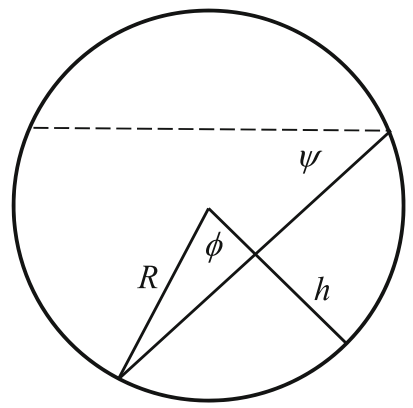

Fig. 1 Schematic of the bed cross-section

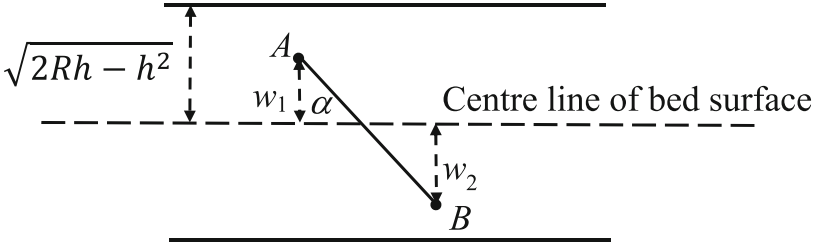

Fig. 2 View of the surface of the bed of particles. The upper and lower solid lines show the intersections of the surface of the bed with the walls of the cylinder. The dashed line showing the centre line of the bed surface is parallel to the axis of the cylinder. Bulk flow is from left to right. The rotation brings particles to the surface in the upper part of the figure and removes particles from the surface in the lower part of the figure

the line of steepest descent, and be reabsorbed into the lower part of the bed at $B$, and there rejoin the rigid body rotation. The avalanche is assumed to be very thin, and the particle is assumed to spend a negligible time in it. These approximations are made to simplify the calculations. The aim of the current work is to demonstrate the effect of the geometry of the avalanches on mixing, and that effect will be emphasised by these approximations. Kohav et al. [31] have proposed a method for taking into account the thickness of the avalanche, and Rogers and Gardner [24] and Kohav et al. [31] have proposed methods for taking into account the time spent in avalanching. Ignoring collisional dispersion, the path of the particle is given by the angle $\alpha$, which is shown in Fig. 2, and is given by (Pickering et al. [20])

$\tan \alpha=\frac{\sin \beta}{\sqrt{\sin ^{2} \gamma-\sin ^{2} \beta}}$

so that the axial distance $\Delta z$ that the particle travels in the avalanche is given by

$\Delta z=\tan \alpha\left(w_{1}+w_{2}\right)$

Here $w_{1}$ and $w_{2}$ are the distances in the bed surface of $A$ and $B$, respectively, from the centreline of the bed surface, and are shown in Fig. 2.

A number $N$ of particles is chosen. Initially, the particles are distributed at random at axial position $z=0$, over the part of the cross section of the cylinder occupied by the bed. As time progresses, the position of each particle is tracked. When a particle in the bed rotates to the free surface, $A$, it is assumed to move immediately to a position $B$ chosen at random on the line of steepest descent on the lower half of the bed surface, after which the particle rotates about the axis without change in axial position.

For simplicity, avalanches are first assumed to be wellmixed, so that in Fig. 2 the position along the line of steepest descent of $B$ is independent of position $A[16,31]$. A comment on a different hypothesis, that $w_{1}=w_{2}$, will be made later. The probability distribution for choosing point $B$ is 
assumed to be proportional to the volumetric rate per unit surface area at which the granular bed is rotated away from the free surface. At distance $w$ from the centreline of the free surface this is

$p(w) \propto n w$

so that $[16,31]$

$p\left(w_{2}\right)=\frac{2 w_{2}}{R^{2}-h^{2}}$

Equivalently, the variable $r$, the distance of $B$ from the axis of the cylinder, that is

$r=\sqrt{(R-h)^{2}+w_{2}^{2}}$

can be used, with

$p(r)=\frac{2 r}{2 R h-h^{2}}$

The assumptions made in this model imply that

$R M S D=\tan ^{2} \alpha R^{2} f(t n)$

where the function $f$ is independent of cylinder inclination, $\beta$, angle of repose, $\gamma$, and radius, $R ; f$ depends on fill. The factor $\tan ^{2} \alpha R^{2}$ arises because $w_{1}$ and $w_{2}$ are proportional to $R$, the constant of proportionality depending on the value of the fill, and the axial distance travelled for a given $w_{1}$ and $w_{2}$ is $\tan \alpha\left(w_{1}+w_{2}\right)$. Now consider the path in space of a single particle, which is independent of rotational speed. At time $t$, the axial position of the particle will depend on the total amount of rotation in the particle bed that the particle has experienced, which is $2 \pi t n$ radians. This means that $z$ is a function of $t n$.

Some sample results for geometric dispersion are now given for the following experimental conditions: $R=0.05$ $\mathrm{m}, n=\frac{1}{12} \mathrm{rps}, \gamma=28^{\circ}, \beta=7.5^{\circ}$, and with $N=10^{5}$. $R M S D$ is shown for fill $=0.26$ in Fig. $3 \mathrm{a}$, and for fill $=0.5$ in Fig. 3b. These conditions were chosen to match the DEM simulations presented in Sect. 3. Both figures display oscillations. These experimental conditions give the Froude number $F r=(2 \pi n)^{2} R / g$, which with fill $f>0.1$ gives the rolling mode with a locally flat bed surface [12]; such behaviour is expected for $10^{-4}<F r<10^{-2}$.

The cause of these oscillations can be demonstrated by the following crude model for the axial motion within an inclined cylinder. Consider a set of particles in the granular bed which all start at axial position $z=0$ and time $t=0$. Suppose that after a period $\tau$ these particles have all moved to axial position $\delta$; at time $t$, where $0<t<\tau$, a fraction $(1-t / \tau)$ of the particles has yet to move down an avalanche

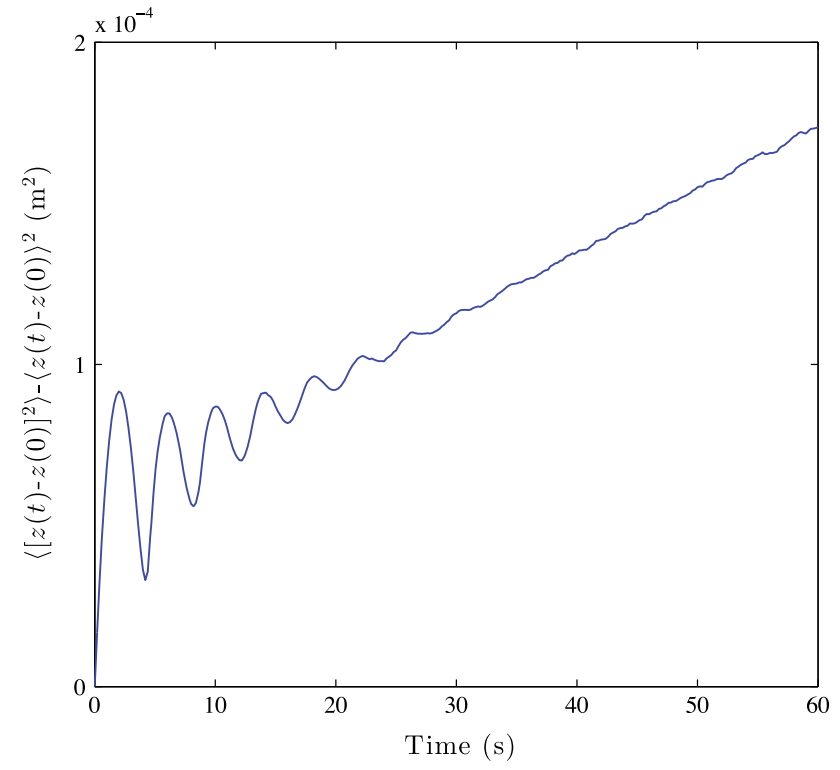

(a)

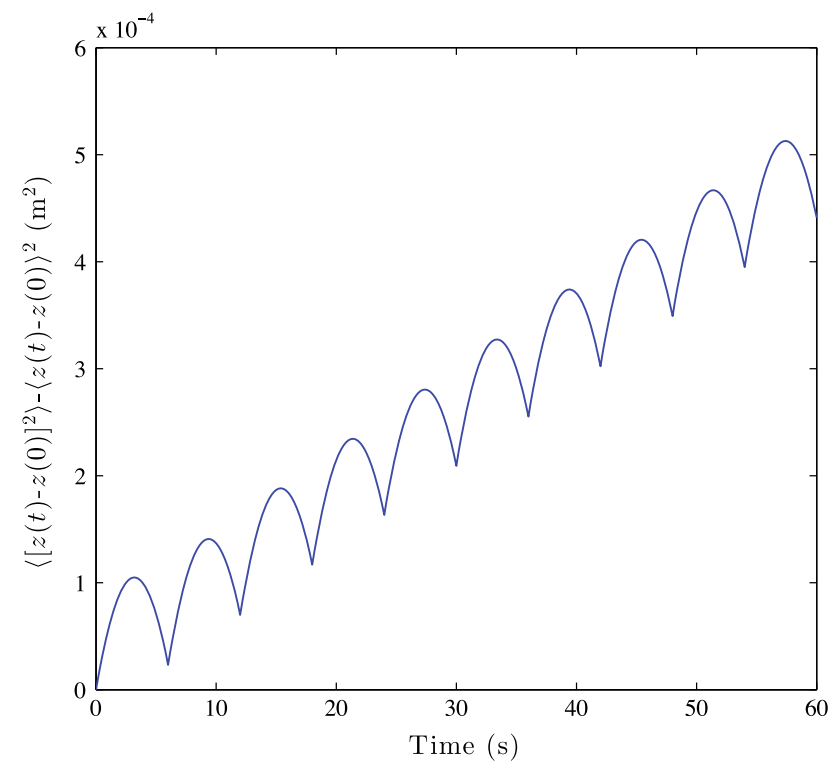

(b)

Fig. $3 R M S D$ predicted by the Monte Carlo model with perfect radial mixing during avalanches and no collisional dispersion. The following parameter values were used in the model: $R=0.05 \mathrm{~m}, n=\frac{1}{12} \mathrm{rps}$, $\gamma=28^{\circ}, \beta=7.5^{\circ}$, and with $N=10^{5}$. a Fill $=0.26$. b Fill $=0.5$

and is still at $z=0$, while a fraction $t / \tau$ has moved to $z=\delta$. Then, during the period $0<t<\tau$, the mean displacement and mean squared displacement are:

$\langle z\rangle=\frac{t}{\tau} \delta,\left\langle z^{2}\right\rangle=\frac{t}{\tau} \delta^{2}$

so RMSD is given by (note that here $z(0)=0$ for all particles)

$R M S D=\left(\frac{t}{\tau}-\frac{t^{2}}{\tau^{2}}\right) \delta^{2}$ 
which varies quadratically with time during the period $0<$ $t<\tau$. If this behaviour continued, there would be oscillations in the magnitude of axial dispersion: at integer values of $t / \tau$ all the particles would be at the same axial position and $R M S D$ would be zero, whereas $R M S D$ would vary parabolically with $t / \tau$ for non-integral $t / \tau$.

Now consider the case of fill $=0.5$. The particles which are initially distributed uniformly over the semicircle at $z=0$ will be denoted tagged particles. After a period $\tau$, which is half the period of rotation of the cylinder, each tagged particle will have rotated to the free surface of the bed, fallen in an avalanche, and then rotated back to its original azimuthal position; each particle will have changed its radial and axial positions during the avalanche. At time $t=0$, the distribution of tagged particles possesses azimuthal symmetry, that is at each radial and axial position the distribution of tagged particles is independent of azimuthal position in the particle bed. This symmetry ensures that throughout the period $0<t<\tau$, the composition of avalanches in terms of tagged particles is constant, and thus the distribution in $z$ of tagged particles which have avalanched is constant. So at time $t$, where $0<t<\tau$, a fraction $(1-t / \tau)$ of the particles will still be at $z=0$, while a fraction $t / \tau$ will have reached their new axial position, and

$$
\langle z(t)\rangle=\frac{t}{\tau}\langle z(\tau)\rangle,\left\langle z^{2}(t)\right\rangle=\frac{t}{\tau}\left\langle z^{2}(\tau)\right\rangle
$$

In this case $R M S D$ varies quadratically with time during the period $0<t<\tau$. But the behaviour differs from the crude model because, in contrast to the crude model which assumes that all particles are displaced the same axial distance $\delta$ by an avalanche, the distance travelled by a particle in an avalanche is selected at random from a distribution by the Monte Carlo model. Since the axial displacement of the particle is proportional to the distance it travels in an avalanche (equation (7)), the axial displacement of a particle during an avalanche follows a distribution, leading to geometric axial dispersion, with $\left\langle z(\tau)^{2}\right\rangle \geq\langle z(\tau)\rangle^{2}$. The same argument for azimuthal symmetry can be made for subsequent periods of time $\tau$, and $R M S D$ increases with time, with oscillations about that increase.

For the case of fill $=0.26$ particles at different radii have different residence times in a period of rigid body rotation, which is different from the case of fill $=0.5$. This means that when some particles have had one avalanche, others have had more than one avalanche, and there are no times at which the distributions of tagged particles show azimuthal symmetry. However, because of the circular geometry, there are relatively more particles at large radius, and the behaviour of these particles can for short times approximate that of the case of fill $=0.5$, giving oscillations. But as time increases any such regularity will be smeared out. Thus the Monte Carlo model predicts that RMSD increases with time, with oscillations that decay with time.

The results shown in Fig. 3a, b have been for geometric axial dispersion only. Collisional axial dispersion has been ignored, which means that if the model were used for a horizontal cylinder there would be no axial dispersion, which is incorrect. Rogers and Gardner [24] and Das Gupta et al. [16] incorporate collisional dispersion in their Monte Carlo models by allowing variation in the angle of descent down the avalanche. Here the same will be done, by incorporating an additional contribution to the axial motion in the form of a Gaussian distribution with width proportional to $\left(w_{1}+w_{2}\right)$. The constant of proportionality is chosen to match the corresponding DEM results for the case of a horizontal cylinder, which will be discussed later. Results are again given for experimental conditions: $R=0.05 \mathrm{~m}, n=\frac{1}{12} \mathrm{rps}$, $\gamma=28^{\circ}, \beta=7.5^{\circ}$, and with $N=10^{5}$. The standard deviation of the Gaussian distribution has been taken to be 0.055 . $R M S D$ is shown for fill $=0.26$ in Fig. $4 \mathrm{a}$, and for fill $=0.5$ in Fig. 4b. The addition of collisional dispersion has led to $R M S D$ increasing in magnitude in both cases. In both cases, the contributions to $R M S D$ from geometric and collisional dispersion are additive.

Kohav et al. [31] and Li et al. [34] used the assumption that the avalanche is not well-mixed, so that $w_{2}$ is correlated with $w_{1}$. Ingram et al. [35] have shown that this is the case in both horizontal and inclined cylinders. As an extreme form of correlation, results from a Monte Carlo model for geometric dispersion with $w_{2}=w_{1}$ are shown in Fig. 5a, b. Both figures show RMSD with oscillations about a quadratic increase with time. Note that this quadratic increase with time does not indicate superdiffusive behaviour, but arises as a consequence of the geometry of the system and the assumption that particles do not mix in the transverse plane, i.e. $w_{2}=w_{1}$. Under these conditions the axial displacement of a particle per avalanche depends on its radial position, but the particle is displaced by the same distance in each avalanche. Consequently, the second central moment of the distribution of axial displacements increases as $t^{2}$.

It is reiterated that the Monte Carlo model is not intended to be realistic, in that important aspects of the dynamics have been ignored, including the thickness and duration of avalanches, and ideal extremes of avalanche mixing have been used. Nevertheless it is hoped that the results demonstrate the consequences of the geometry of successive avalanches and are illustrative of possible behaviour. DEM modelling, which does not make such simplifications, now follows. 


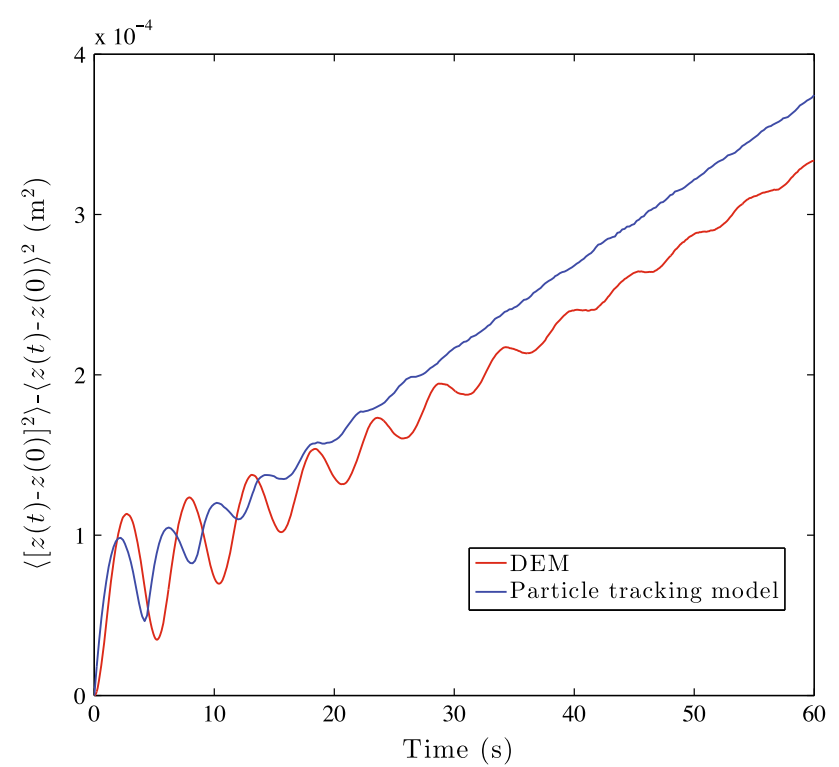

(a)

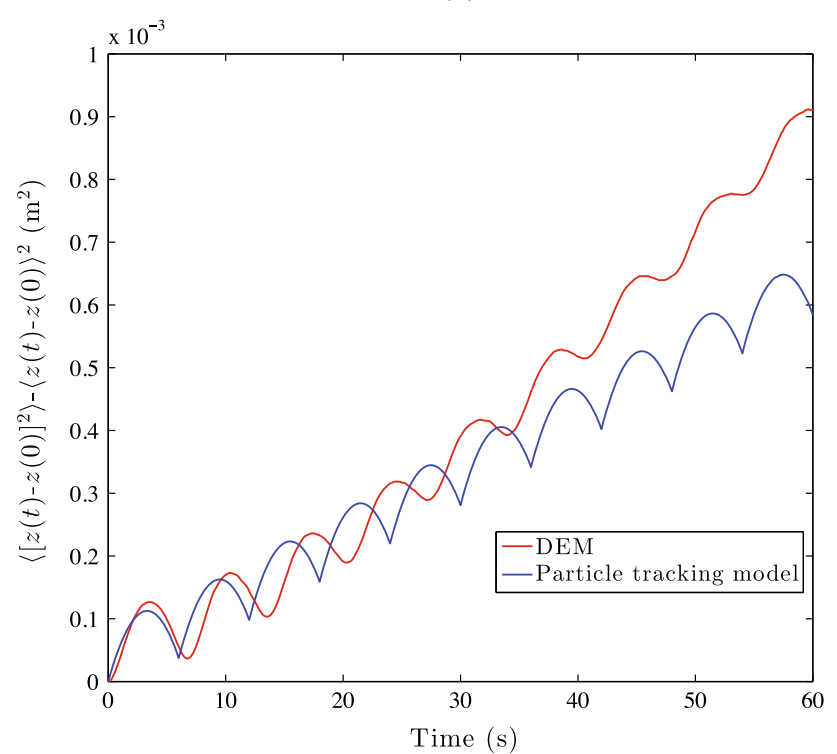

(b)

Fig. $4 R M S D$ predicted by the Monte Carlo model with perfect radial mixing and collisional dispersion during avalanches. The following values parameter values were used in the model: $R=0.05 \mathrm{~m}, n=\frac{1}{12} \mathrm{rps}$, $\gamma=28^{\circ}, \beta=7.5^{\circ}$, and with $N=10^{5}$. Data from DEM simulations of beds composed of $3 \mathrm{~mm}$ spheres, discussed in Sect. 3, are also shown. a Fill $=0.26$. $\mathbf{b}$ Fill $=0.5$

\section{Discrete element method simulations}

Simulations of the system described above have been performed using the soft-sphere DEM [36]. The implementation of the DEM employed here is described by Third et al. [18]. A linear spring and dashpot model was used to describe contacts between particles and attractive forces between particles were prevented. Hence the contact force in the direction normal to the particle surfaces was given by:

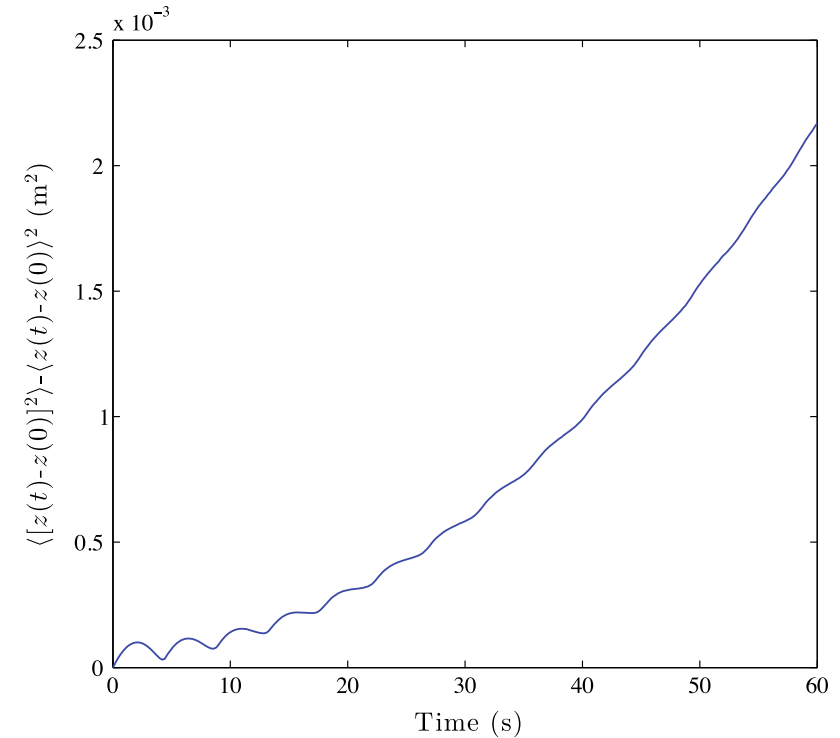

(a)

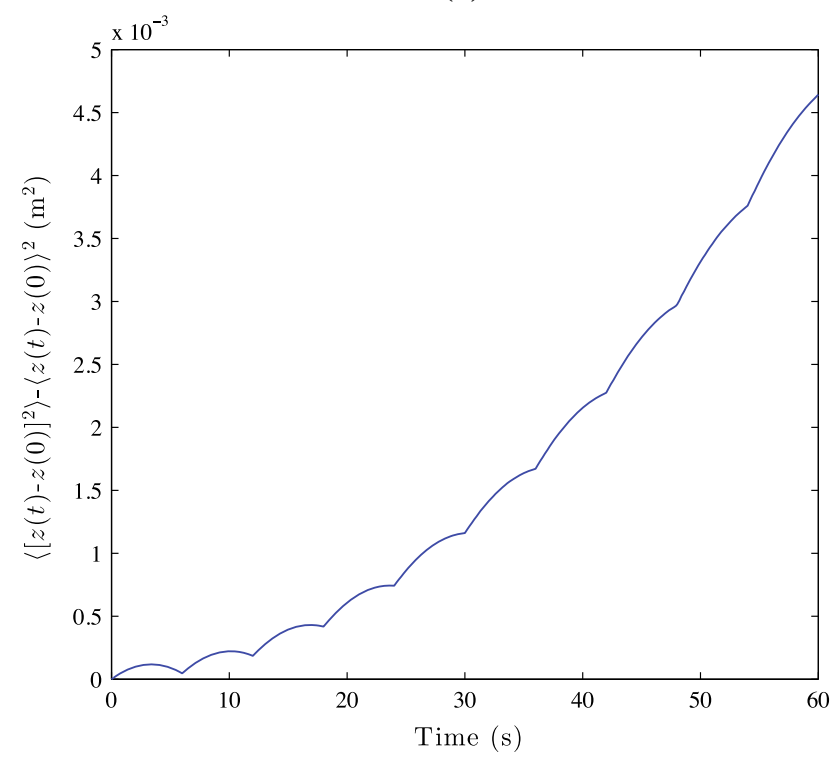

(b)

Fig. 5 RMSD predicted by the Monte Carlo model with no radial mixing during avalanches. The following values parameter values were used in the model: $R=0.05 \mathrm{~m}, n=\frac{1}{12} \mathrm{rps}, \gamma=28^{\circ}, \beta=7.5^{\circ}$, and with $N=10^{5}$. a Fill $=0.26$. b Fill $=0.5$

$F_{n}=\max \left(0, k_{n_{i j}} \delta_{n}-2 \eta_{n} \sqrt{m_{i j} k_{n_{i j}}} v_{n}\right)$

Here $\delta_{n}$ is the overlap between the contacting particles, $\eta_{n}$ is the damping factor in the normal direction, $v_{n}$ is the relative velocity in the normal direction, $m_{i j}$ is the effective mass given by $1 / m_{i j}=1 / m_{i}+1 / m_{j}$ and $k_{n_{i j}}$ is the effective normal stiffness defined as $1 / k_{n_{i j}}=1 / k_{n_{i}}+1 / k_{n_{j}}$. In the tangential direction, the magnitude of the friction force was restricted by Coulomb's law, such that:

$F_{t}=\min \left(\mu k_{n_{i j}} \delta_{n}, k_{t_{i j}} \delta_{t}-2 \eta_{t} \sqrt{m_{i j} k_{n_{i j}}} v_{t}\right)$ 
Here $\mu$ is the coefficient of friction, $\eta_{t}$ is the damping factor in the tangential direction and $v_{t}$ is the relative velocity in the tangential direction. The tangential displacement, $\delta_{t}$, is defined as $\int v_{t} d t$ and the effective tangential stiffness was calculated as $1 / k_{t_{i j}}=1 / k_{t_{i}}+1 / k_{t_{j}}$ The equations of motion are integrated using a third order Adams-Bashforth scheme with a timestep $d t$ which satisfies by $d t \leq t_{\text {col }} / 30$, where $t_{\mathrm{col}}$ is the duration of a binary collision. Table 1 shows the parameter values used in the DEM simulations reported here. These parameter values were chosen to match the values used by Third et al. [18], who reported that the axial dispersion coefficient of $3 \mathrm{~mm}$ spheres does not depend strongly on the values used for the spring stiffnesses or the damping factors.

A particle size distribution of $\pm 5 \%$ relative to the nominal particle diameter was introduced to prevent particle crystallisation effects. Wall rougheners were used to suppress slip between the spheres and the cylindrical wall (see Fig. 6). The

Table 1 Parameter values used in the DEM simulations

\begin{tabular}{lll}
\hline Name & Symbol & Value \\
\hline Nominal particle diameter & $d$ & $3.0 \times 10^{-3} \mathrm{~m}$ \\
Particle size distribution & - & $\pm 5 \%$ \\
Particle density & $\rho$ & $2500 \mathrm{~kg} / \mathrm{m}^{3}$ \\
Normal spring stiffness & $k_{n}$ & $2000 \mathrm{~N} / \mathrm{m}$ \\
Tangential spring stiffness & $k_{t}$ & $1000 \mathrm{~N} / \mathrm{m}$ \\
Normal damping factor & $\eta_{n}$ & 0.22 \\
Tangential damping factor & $\eta_{t}$ & 0.2 \\
Coefficient of friction & $\mu$ & 0.7 \\
Acceleration due to gravity & $g$ & $9.81 \mathrm{~m} / \mathrm{s}^{2}$ \\
Angle of inclination & $\beta$ & $7.5^{\circ}$ \\
Radius of the cylinder & $R$ & $0.05 \mathrm{~m}$ \\
Periodic cell length & $L$ & $0.21 \mathrm{~m}$ \\
Rotation speed of the cylinder & $n$ & $\frac{1}{12} \mathrm{rps}$ \\
Number of particles & $N_{p}$ & 15360,31460 \\
Timestep for the DEM & $d t$ & $1.0 \times 10^{-5} \mathrm{~s}$ \\
\hline
\end{tabular}

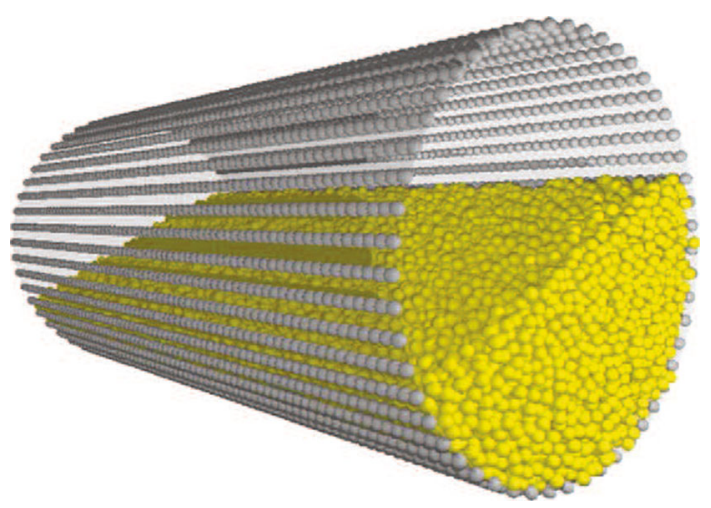

Fig. 6 Snapshot from a DEM simulation of a drum containing 31460 particles. The particles on the cylinder wall are wall rougheners wall rougheners were made of lines of $3 \mathrm{~mm}$ spheres, running along the axial direction of the cylinder. The centres of these spheres were located on the surface of the cylinder, leading to an uneven surface that hinders relative motion between the bed of spheres and the cylinder wall. In total 52 equally spaced wall rougheners were positioned around the circumference of the cylinder. To allow a continuous flow of particles through the cylinder to be modelled, periodic boundary conditions (PBCs) were applied along the axial direction. The length of the periodic cell was chosen to be $L=0.21$ $\mathrm{m}$. The angle of repose of the simulated bed was found to be $\gamma=28^{\circ}$. Simulations of a system with $L=0.105 \mathrm{~m}$ have also been performed and were found to give very similar results to those presented here. It has been shown that the results are independent of $L$ for $L / d=70$ and 35. While this is encouraging, further work is needed to demonstrate that the results are independent of $L$ for $L / d>70$, and for other parameter values.

To evaluate RMSD from the DEM data, $900 \mathrm{~s}$ of simulated time was divided into segments of $60 \mathrm{~s}$. For each time segment $R M S D$ was calculated as a function of time, from the start of the time segment, based on the position of every particle. Here the average $R M S D$ for the 15 different $60 \mathrm{~s}$ time segments is reported. This averaging process helps to reduce the effect of random fluctuations arising from the small number of particles in the simulated system. To ensure that the data reported corresponds to the steady state of the system, the cylinder was rotated for $40 \mathrm{~s}$ prior to the start of the first time segment used in the calculation of $R M S D$.

Figure $4 \mathrm{a}, \mathrm{b}$ show $R M S D$ calculated from DEM simulations for fill levels of 0.26 and 0.5 , respectively. For the case of fill $=0.26$ there is good qualitative agreement between the DEM data and the Monte Carlo model. For both models RMSD oscillates about a linear increase with time for small times, and at larger times both models predict that the oscillation is damped out. Furthermore, the period and initial amplitude of the oscillations shown in Fig. 4a are comparable. However, the Monte Carlo model predicts that the amplitude of the oscillations decreases more quickly than is observed in the DEM data. It is believed that this discrepancy arises because the Monte Carlo model ignores the residence time in avalanching and the thickness of the avalanching layer and assumes perfect mixing within the avalanches.

For fill $=0.5$ the DEM predicts that $R M S D$ oscillates about a line whose gradient increases with time. This behaviour does not agree with the Monte Carlo model with prefect mixing and appears to share some characteristics with the model with no mixing (Fig. 5b). This observation suggests that the rate of mixing in the transverse plane is lower for the fill $=$ 0.5 case. This explanation is in agreement with previously reported results for the influence of fill level on transverse mixing within rotating cylinders [37,38]. Carley-Macauly and Donald [37] studied the mixing of Bedford sand in a hor- 
izontal cylinder with a diameter of $101.6 \mathrm{~mm}$ and a length of $203.2 \mathrm{~mm}$ and found that the mixing time increased rapidly for fill levels approaching 0.5. Hogg and Fuerstenau [38] mixed quartz particles in a horizontal cylinder with diameter $140 \mathrm{~mm}$ and length $76.2 \mathrm{~mm}$ and reported that the mixing efficiency for a fill of 0.5 was substantially lower than for a fill of 0.3 .

The different mixing rates of the two fill levels may be demonstrated from the DEM data by considering the mean axial displacement of the particles as a function of location in the transverse plane. The spatially resolved mean axial displacements after a time of $200 \mathrm{~s}$ are shown in Fig. 7. These data were obtained by dividing the transverse plane into square bins with a side length of $4 \mathrm{~mm}$. Data for fill = 0.26 are in Fig. 7 a, which shows that the mean axial displacement of the particles is approximately constant throughout the transverse plane. Data for fill $=0.5$, Fig. $7 \mathrm{~b}$, show that in contrast, a clear spatial dependence can be observed for the mean axial displacement of particles in the fill $=0.5$ case. Figure $7 \mathrm{~b}$ shows that, on average, particles located close to the cylinder wall during rigid body rotation (i.e. particles with large $r$ ) have larger axial displacements than particles located in the centre of the bed. This phenomenon occurs because the axial displacement of a particle is proportional to the distance it travels on the free surface, Eq. (7). Particles located closer to the cylinder wall travel further along the free surface during an avalanche and, because transverse mixing is poor for fill $=0.5$, these particles remain close to the cylinder wall over several avalanches.

\section{Conclusions and future work}

Monte Carlo models have been used to examine the dispersion of particles in inclined rotating cylinders. These models predict that the mean square displacement relative to the mean displacement of the bed undergoes oscillations, which have not previously been reported. The behaviour of the system was found to depend on the fill level of the cylinder and the assumptions made about mixing in avalanches. For the case that particles do not mix during avalanches, $R M S D$ was found to oscillate about a quadratic increase with time, whereas for perfect mixing the oscillations were found to occur about a linear increase with time. For low fill levels these oscillations damp out with time, while undamped oscillations are predicted for a fill level of 0.5. DEM simulations demonstrated good qualitative agreement with the Monte Carlo model with perfect mixing for the fill $=0.26$ case. For fill $=0.5$ the DEM data showed qualitative agreement with the un-mixed Monte Carlo model. This finding is in agreement with Carley-Macauly and Donald [37] and Hogg and Fuerstenau [38], both of whom reported that the mixing time increases dramatically for fill levels close to 0.5 .

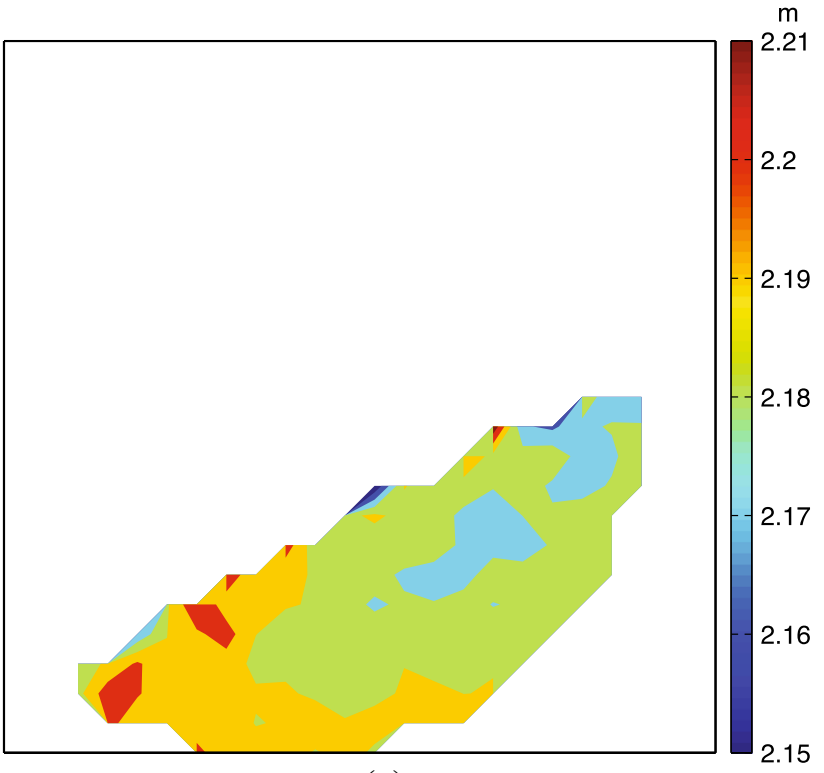

(a)

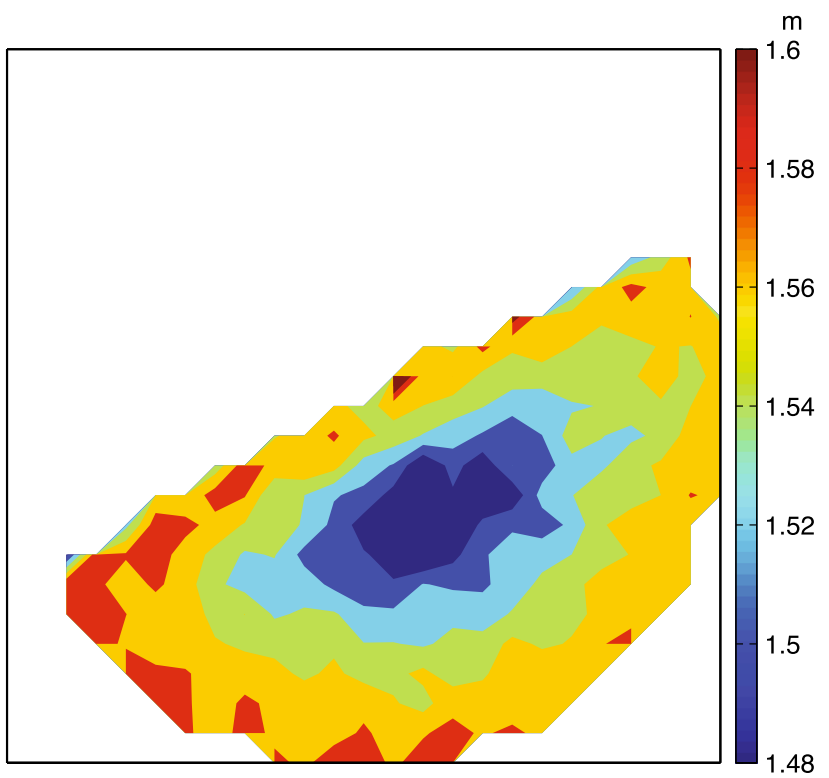

(b)

Fig. 7 Mean axial displacement of the particles as a function of position in the cross-section of the bed. The displacements were measured for an observation time of $200 \mathrm{~s}$ and the bed cross-section was divided into $4 \mathrm{~mm}$ square bins. a Fill $=0.26$. b Fill $=0.5$

The results in Fig. 4 suggest that for $f=0.26$ the oscillations decay after $\mathrm{O}(10)$ periods, and that for $f=0.5$ the amplitude of the oscillations is small compared to the mean RMSD after $\mathrm{O}(10)$ periods. While the oscillations indicate poor mixing at early times, their effect is unlikely to be important for industrial processes where a typical particle experiences many cycles within the equipment.

Further work is planned to investigate the following: (i) The influence of wall roughening, in particular whether the 
oscillations described here are still present in the case in which the walls are not artificially roughened. (ii) The independence of the phenomenon on $L$ for a wider range of parameter values. (iii) The influence of varying fill. (iv) The significance of geometric dispersion compared to collisional dispersion. (v) The oscillations for $f=0.5$.

Acknowledgments We are grateful to the referees for helpful and constructive comments.

\section{References}

1. Jaeger, H.M., Nagel, S.R., Behringer, R.P.: Rev. Mod. Phys. 68, 1259 (1996)

2. Hill, K.M., Khakhar, D.V., Gilchrist, J.F., McCarthy, J.J., Ottino, J.M.: Proc. Natl. Acad. Sci. 96, 11701 (1999)

3. Müller, C.R., Davidson, J.F., Dennis, J.S., Fennell, P.S., Gladden, L.F., Hayhurst, A.N., Mantle, M.D., Rees, A.C., Sederman, A.J.: Phys. Rev. Lett. 96, 154504 (2006)

4. Aranson, I.S., Tsimring, L.S.: Rev. Mod. Phys. 78, 641 (2006)

5. Sandnes, B., Flekkøy, E.G., Knudsen, H.A., Måløy, K.J., See, H.: Nat. Comm. 2, 288 (2011)

6. Seiden, G., Thomas, P.J.: Rev. Mod. Phys. 83, 1323 (2011)

7. Ottino, J.M., Khakhar, D.V.: Annu. Rev. Fluid Mech. 32, 55 (2000)

8. Fischer, R., Gondret, P., Rabaud, M.: Phys. Rev. Lett. 103, 128002 (2009)

9. Ristow, G.H.: Europhys. Lett. 34, 263 (1996)

10. Hajra, S.K., Khakhar, D.V.: Phys. Fluids 17, 013101 (2005)

11. Zik, O., Stavans, J.: Europhys. Lett. 16, 255 (1991)

12. Mellmann, J.: Powder Technol. 118, 251 (2001)

13. Hehl, M., Kröger, H., Helmrich, H., Schügerl, K.: Powder Technol. 20, 29 (1978)

14. Third, J.R., Müller, C.R.: Phys. Rev. E 86, 061314 (2012)

15. Hogg, R., Cahn, D.S., Healy, T.W., Fuerstenau, D.W.: Chem. Eng. Sci. 21, 1025 (1966)
16. Das Gupta, S., Khakhar, D.V., Bhatia, S.K.: Powder Technol. 67, 145 (1991)

17. Taberlet, N., Richard, P.: Phys. Rev. E 73, 041301 (2006)

18. Third, J.R., Scott, D.M., Scott, S.A.: Powder Technol. 203, 510 (2010)

19. Christov, I.C., Stone, H.A.: Proc. Natl. Acad. Sci. 109, 16012 (2012)

20. Pickering, R.W., Feakes, F., Fitzgerald, M.L.: J. Appl. Chem. 1, 13 (1951)

21. Saeman, W.C.: Chem. Eng. Prog. 47, 508 (1951)

22. Vàhl, L., Kingma, W.: Chem. Eng. Sci. 1, 253 (1952)

23. Kramers, H., Croockewit, P.: Chem. Eng. Sci. 1, 259 (1952)

24. Rogers, R.S.C., Gardner, R.P.: Powder Technol. 23, 159 (1979)

25. Rao, S.J., Bhatia, S.K., Khakhar, D.V.: Powder Technol. 67, 153 (1991)

26. Scott, D.M., Davidson, J.F., Lim, S.-Y., Spurling, R.J.: Powder Technol. 182, 466 (2008)

27. Rapaport, D.C.: Phys. Rev. E 65, 061306 (2002)

28. Third, J.R., Scott, D.M., Scott, S.A., Müller, C.R.: Granul. Matter 13, 75 (2011)

29. Gao, Y., Glasser, B.J., Ierapetritou, M.G., Cuitino, A., Muzzio, F.J., Beeckman, J.W., Fassbender, N.A., Borghard, W.G.: AIChE J. 59, 4068 (2013)

30. Campbell, C.S.: J. Fluid Mech. 348, 85 (1997)

31. Kohav, T., Richardson, J.T., Luss, D.: AIChE J. 41, 2465 (1995)

32. Davidson, J.F., McTait, G.E., Scott, D.M.: In: Fan, L.S., Knowlton, T. (eds.) Fluidization IX: Proceedings of the ninth engineering foundation conference on fluidization, pp. 397-414. Engineering Foundation, Durango (1998)

33. McTait, G.E.: Residence times and solid flows in rotary kilns. Ph.D. thesis, University of Cambridge (1998)

34. Li, S.Q., Yan, J.H., Li, R.D., Chi, Y., Cen, K.F.: Powder Technol. 126, 217 (2002)

35. Ingram, A., Seville, J.P.K., Parker, D.J., Fan, X., Forster, R.G.: Powder Technol. 158, 76 (2005)

36. Cundall, P.A., Strack, O.D.L.: Géotechnique 29, 47 (1979)

37. Carley-Macauly, K.W., Donald, M.B.: Chem. Eng. Sci. 19, 191 (1964)

38. Hogg, R., Fuerstenau, D.W.: Powder Technol. 6, 139 (1972) 